\title{
High Body Mass Index and Thyroid Stimulating Hormone Levels Do Not Affect Thyroid Nodule Selection for Fine-Needle Aspiration Biopsy after Ultrasound Evaluation
}

\author{
Hyun Gi Kim ${ }^{1,2, *}$, Hye Sun Lee ${ }^{3}$, Eun Kyung Kim², Chung-Mo Nam ${ }^{2}$, \\ Hee Jung Moon', Hae Kyoung Jung ${ }^{5}$ and Jin Young Kwak ${ }^{2}$ \\ Department of Radiology, Ajou University Medical Center, Ajou University School of Medicine ${ }^{1}$, Suwon, Department of Radiology, \\ Research Institute of Radiological Science, Yonsei University College of Medicine ${ }^{2}$, Biostatistics Collaboration Unit, Medical Research \\ Center, Yonsei University College of Medicine, Department of Preventive Medicine, Yonsei University College of Medicine ${ }^{4}$, Seoul, \\ Department of Radiology, CHA Bundang Medical Center, CHA University School of Medicine, Seongnam, Korea
}

Background and Objectives: This study was to evaluate whether high body mass index (BMI) or thyroid stimulating hormone (TSH) level would affect selecting thyroid nodule for fine-needle aspiration biopsy (FNA) after ultrasound (US) evaluation. Materials and Methods: A total of 3155 thyroid nodules (2159 benign and 996 malignant nodules) were included. Four grades of BMl and three levels of TSH were applied for grouping. US features of the thyroid nodules were divided into 'probably benign' and 'suspicious for malignancy' categories. Patients were grouped according to gender and univariate and multivariate logistic regression analysis were used to find the association between variables and malignancy. Results: TSH levels were significantly higher in the malignant group $(p<0.001)$. The grades of BMl did not show difference between the malignant and benign groups (females, $p=0.074$ and males, $p=0.157$ ). Younger age and 'suspicious for malignancy' US category were independent risk factors for malignancy in both genders. In females, a high TSH level (odds ratio=1.010, $p<0.001$ ) had significant association with malignancy. Except for younger age (odds ratio $=0.998$, $\mathrm{p}<0.001$ ), no variable in nodules with 'probably benign' US category was significantly associated with malignancy. Conclusion: High TSH levels were more frequent in thyroid malignancy group, but neither high BMI nor high TSH level give additional information for FNA selection after US.

Key Words: Thyroid cancer, Ultrasound, Obesity, Thyroid stimulating hormone, Fine-needle aspiration biopsy

\section{Introduction}

Thyroid cancer incidence has been sharply increasing worldwide during the last few decades. ${ }^{1,2)}$ This is partially due to increased medical surveillance and the widespread use of highly sensitive diagnostic tools such as ultrasound (US) and fine-needle aspiration biopsy (FNA).' However, the increased incidence of large papillary thyroid cancer cannot be explained solely by these reasons. ${ }^{3)}$

There are many potential risk factors for thyroid cancer such as ionizing radiation, history of thyroid cancer in first degree relatives, prior history of thyroid

Received October 15, 2018 / Revised lst November 22, 2018, 2nd March 12, 2019 / Accepted April 29,2019 Correspondence: Jin Young Kwak, MD, PhD, Department of Radiology, Research Institute of Radiological Science, Yonsei University College of Medicine, 50 Yonsei-ro, Seodaemun-gu, Seoul 03722, Korea

Tel: 82-2-2228-7413, Fax: 82-2-393-3035, E-mail: docjin@yuhs.ac

*Current affiliation: Department of Radiology, Eunpyeong St. Mary's Hospital, College of Medicine, The Catholic University of Korea, Seoul, Korea

Copyright (c) 2019, the Korean Thyroid Association. All rights reserved

() This is an open-access article distributed under the terms of the Creative Commons Attribution Non-Commercial License (http://creativecommons.org/licenses/by-nc/4.0/), which permits unrestricted non-commercial use, distribution, and reproduction in any medium, provided the original work is properly cited. 
cancer, and a high calcitonin level. ${ }^{4,5)}$ Recently, attention has been drawn toward obesity, which may be another potential risk factor for thyroid cancer, as there was a dramatic increase in the prevalence of both thyroid cancer and obesity during the similar period. ${ }^{3,6)}$ Up to the present, the best level of evidence has been derived from a pooled analysis of prospective studies ( $n=848,932)$ showing that obesity is an independent risk factor for thyroid cancer (hazard ratio: 1.21 in men and 1.16 in women). ${ }^{7)}$ This was also true in a health checkup setting with 15068 subjects and with FNA results of 1427 patients; the prevalence of thyroid cancer in women was associated with a high body mass index (BMI), per $5 \mathrm{~kg} / \mathrm{m}^{2}$ increase (odds ratio $[\mathrm{OR}], 1.63 ; \mathrm{p}<0.001){ }^{8)}$ Along with high $\mathrm{BMI}, \mathrm{a}$ high thyroid stimulating hormone (TSH) level was reported to be a risk factor for malignant thyroid nodules. ${ }^{9-11)}$ There was a positive association between BMI and TSH levels in a study that included euthyroid patients $^{12,13)}$ as well as hypothyroidism patients. ${ }^{14)}$

Although association between thyroid cancer and high BMI or high TSH level has been reported, risk stratification of thyroid nodules detected on US follows the US feature of nodules. Therefore, the most important factor that determines the need for FNA is US features of a thyroid nodule, not BMI or TSH level. None of the previous studies have evaluated the association of high BMI or TSH levels with thyroid malignancy after adjusting for US features and it remains unclear whether these two potential risk factors would affect nodule selection for FNA. Therefore, the purpose of this study was to evaluate whether high BMI or TSH levels would affect the selection of thyroid nodules for FNA after US evaluation, an essential procedure for choosing an adequate treatment plan.

\section{Materials and Methods}

This retrospective study was approved by the institutional review board, and informed consent was waived. Informed consent for US-guided FNA was obtained from all patients prior to each biopsy.

\section{Study Population}

Among thyroid nodules that were biopsied in our institution from February 2006 through December 2008 (13,182 nodules in 12,028 patients), we selected 3698 nodules with the following criteria; nodules $5 \mathrm{~mm}$ or larger, nodules found in patients without thyroid hormone medication history, and those found in patients with information on height, weight, and TSH values available on medical records within three months from the day of FNA. Among the biopsied nodules, 3954 nodules were operated. Five hundred forty three nodules in 536 patients were excluded due to a lack of definitive cytologic or further pathologic diagnosis following surgery. In the study period, cytological reports were categorized as 'benign', 'indeterminate', 'suspicious for papillary thyroid carcinoma', 'malignant', or 'nondiagnostic'. ${ }^{15-18)}$ Definitive cytologic results for analysis included 'benign' and 'malignant'. We excluded 230 nodules (42.4\%) with nondiagnostic, 95 (17.5\%) nodules with indeterminate, and 218 (40.1\%) nodules with suspicious for malignancy cytologic results. Finally, 3155 nodules in 3005 patients were included as the study population.

\section{Data Acquisition and Patient Grouping}

Patients were grouped according to gender. Clinical information was systematically summarized and collected from electronic medical records by a $4^{\text {th }}$ year resident and a trained employee who had worked in our radiology department for 5 years. The patients included in this study had records available of their height, weight, and serum TSH levels for each day of their outpatient clinic visits within three months from the day of FNA. The BMI was calculated (weight $(\mathrm{kg}) /$ height $\left(\mathrm{m}^{2}\right)$ ) using each patient's height and weight. Patients were assigned four grades according to BMI using the World Health Organization classification; underweight with BMI <18.5; normal with BMI 18.5 to 24.9; overweight with BMI 25 to 29.9, and obese with $\mathrm{BMI} \geq 30$. $^{19)}$ Our institution's TSH assay method altered from radioimmunoassay to IRMA method during the study period. Therefore, TSH levels were determined by radioimmunoassay (Trinity Biotech, Co. 


\section{BMI and TSH for Thyroid Nodule FNA}

Wicklow, Ireland, reference range 0.4-3.1 $\mu \| \mathrm{U} / \mathrm{mL}$ ) or IRMA (TSH-CTK-3, SORIN Biomedica, Saluggia, Italy, reference range $0.3-4.99 \mu \mathrm{U} / \mathrm{mL}$ ). The reference ranges of the two TSH level measurement methods were based on each company's recommendation. TSH values were classified into three levels, low (lower than reference range), normal (within reference range), and high (higher than reference range).

\section{Ultrasound Evaluation and FNA}

Images of US were obtained using 5-12 $\mathrm{MHz}$ linear transducers (HDI 5000 and $U$-22, respectively; Philips, Bothell, WA, USA). Ultrasonographic features of the thyroid nodules that underwent US-guided FNA were recorded according to internal component, echogenicity, margin, calcification, shape, and vascularity (Appendix). Suspicious US features include marked hypoechogenicity, microlobulated or irregular margin, microcalcification, taller than wide shape. Nodules are considered 'suspicious for malignancy' if there is one or more of the above suspicious US features. Nodules with no suspicious US features are considered as 'probably benign'. Based on US features, thyroid nodules were divided into two categories, 'probably benign' and 'suspicious for malignancy'. ${ }^{20)}$

All FNA on thyroid nodules was done under US guidance. For FNA, a 23-gauge needle attached to a 2-mL disposable plastic syringe was used. In each nodule, aspiration was done twice or more and aspirated materials were expelled onto glass slides followed by smearing. All smeared glass slides were placed in 95\% alcohol for Papanicolaou staining and the remaining material was rinsed in saline solution. After the glass slides of expelled aspirated material were moved to the pathologic department, they were interpreted by five experienced cytopathologists.

\section{Statistical Analysis}

Two sample t-tests were used to compare continuous variables including age, nodule size, height, and weight of patients in the malignant and benign nodule groups. The Chi-square test was used to compare categorical variables including BMI grade,
TSH level, and US category in the two groups.

The OR with 95\% confidence intervals (CI) of malignancy for each clinical and US feature was calculated with univariate and multivariate logistic regression analysis. As some previous studies showed a positive association between BMI and TSH levels, we analyzed their association. ${ }^{13,21)}$ We evaluated the association between BMI and TSH levels (both in multimodal distribution) by using cumulative logit link and it was not significant ( $p=0.318$ ). Therefore, we included both BMl value and TSH level as independent variables in multivariate logistic regression analysis. After adjusting for all factors in the univariate analysis except for height and weight, which were used to calculate BMI, multivariate logistic regression analysis was performed to assess independent associations of thyroid malignancy with all clinical factors and US categories. To evaluate the effect of clinical factors on nodule selection for FNA, univariate and multivariate logistic regression analysis was done for nodules of each US category. We used logistic regression with GEE (generalized estimating equation) for clustered data. SAS ver. 9.2 (SAS Inc., Cary, NC, USA) was used for statistical analysis. A p value of less than 0.05 was considered significant for statistical analysis.

\section{Results}

Among 3155 nodules, 2663 nodules were in female and 492 nodules were in male. Among the nodules, there were 2159 benign and 996 malignant nodules. There were 2088 patients with benign nodules; 2017 patients had one benign nodule and 71 patients had two benign nodules. There were 933 patients with malignant nodules; 870 patients had one malignant nodule and 63 patients had two malignant nodules. Sixteen patients had both malignant and benign nodules; 15 patients had one malignant and one benign nodule and one patient had two malignant and one benign nodule.

The number of nodules with each clinical and US feature is shown in Table 1. Patients with malignant nodules were significantly younger compared to patients with benign nodules in both genders $(p<0.001)$. 

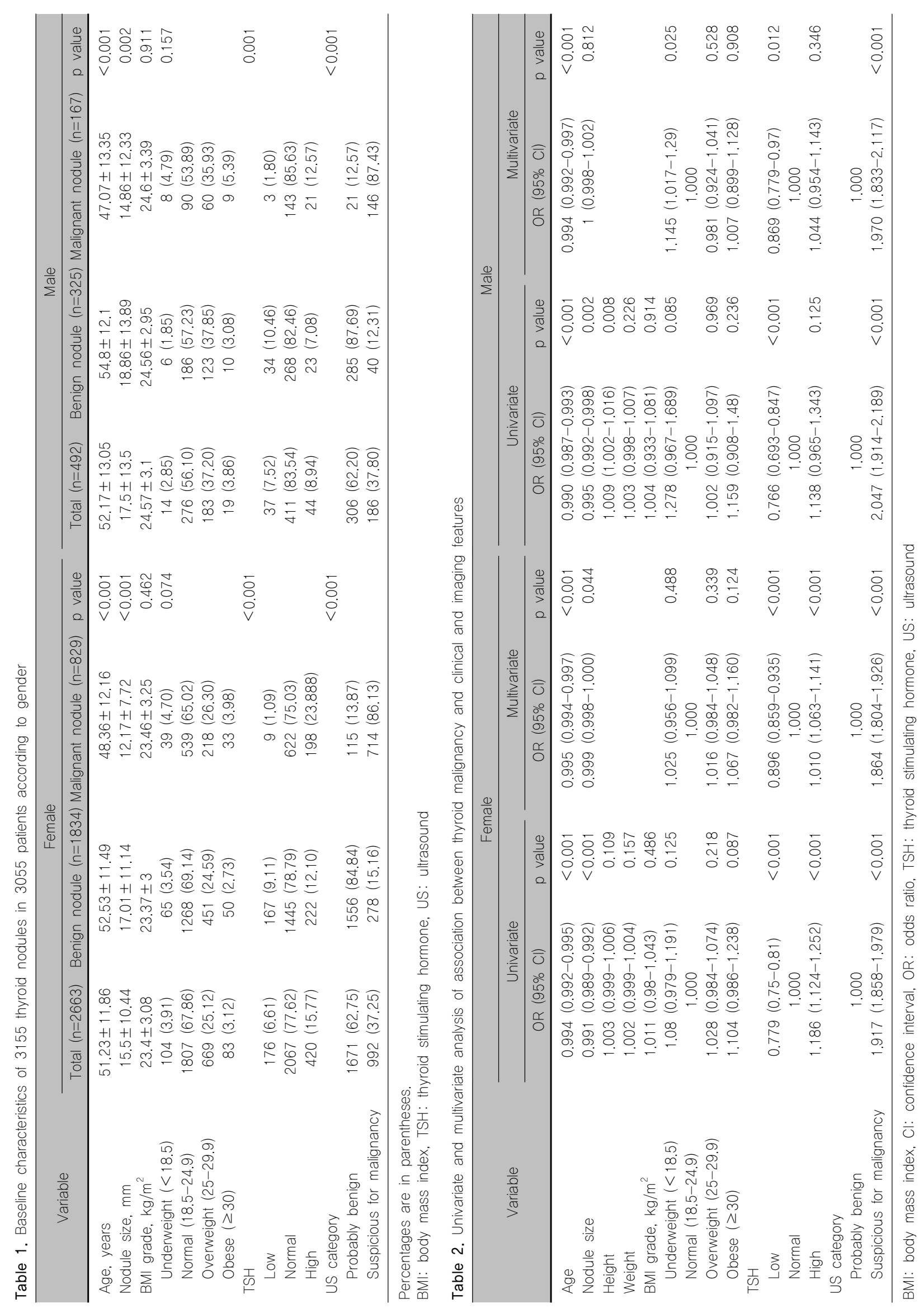


\section{BMI and TSH for Thyroid Nodule FNA}

Malignant nodules had significantly smaller size in both genders $(p<0.001)$. In both genders, the grades of $\mathrm{BMI}$ showed no significant difference between the malignant and benign groups (female, $p=0.074$; male, $p=0.157)$. TSH levels were significantly different between the malignant and benign groups in both genders $(p<0.001)$ showing a higher percentage of high TSH levels among the malignant group (12.6-23.9\%) compared to the benign group (7.1-12.1\%). The US 'suspicious for malignancy' category was significantly more frequent in the malignant group compared to the benign group in both genders $(p<0.001)$.

In both genders, younger age and 'suspicious for malignancy' US category was significantly associated with malignancy on multivariate logistic regression analysis (Table 2). In females, a high TSH level $(\mathrm{OR}=1.010, \quad \mathrm{p}<0.001)$ and smaller nodule size (OR=0.999, $p=0.044)$ also had significant association with malignancy on multivariate analysis. In males, low BMI (underweight; OR=1.145, $p=0.025$ ) was significantly associated with malignant thyroid nodules on multivariate analysis. In both genders, a low TSH level was significantly associated with decreased risk of malignancy (OR=0.766-0.896, $\mathrm{p}<0.001-0.012$ ).

The results of multivariate logistic regression analysis for each US category are shown in Table 3. In 'probably benign' US category, younger age was significantly associated with malignancy in both genders. In females, smaller nodule was significantly associated with malignancy in 'probably benign' US category. In nodules with 'suspicious for malignancy' US category, younger age was significantly associated with malignancy in both genders.

Regarding BMI grade, it did not show any significant association with malignancy in females in both US categories. In males, low BMl was significantly associated with malignancy among nodules with 'suspicious for malignancy' US category. Regarding TSH levels, high TSH level was significantly associated with malignancy in females in 'suspicious for malignancy' US category, but not in males. In both genders, low TSH level was significantly associated with decreased risk of malignancy in 'suspicious for malignancy' US category.

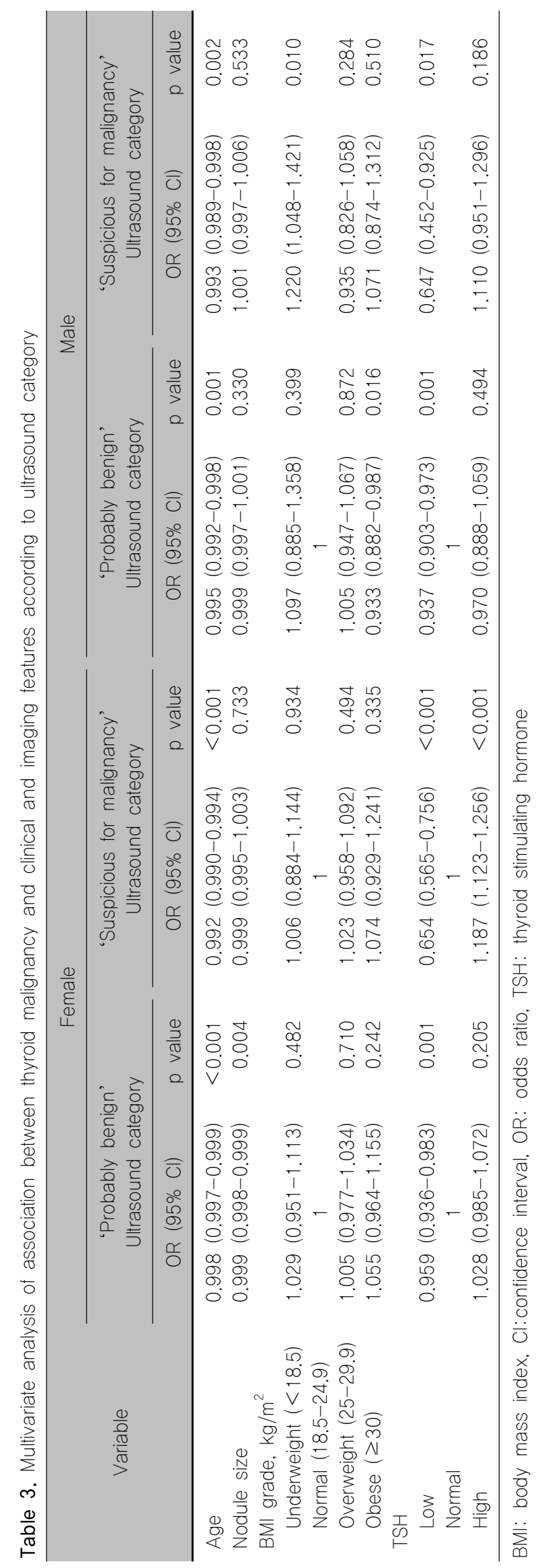




\section{Discussion}

In this study, malignant thyroid nodules were significantly associated with 'suspicious for malignancy' US category in both genders on multivariate analysis. In terms of TSH and BMI, there were different results according to the gender on multivariate analysis. High $\mathrm{TSH}$ levels in females and low BMI grades in males were significantly associated with malignancy. However, when we evaluated the association of thyroid malignancies according to US category, neither a BMI grade nor a TSH level was significantly associated with malignancy in nodules with the 'probably benign' US category. On the other hand, a high TSH level in females and a low BMI grade in males were significantly associated with malignancy in nodules with the 'suspicious for malignancy' US category. Therefore, neither a BMI grade nor TSH levels have additional value in the selection of thyroid nodules for FNA after adequate categorization with US. These results are important because, to date, although the significant associations between malignancy and BMI or TSH levels have been studied, ${ }^{7-10)}$ there has been no study showing whether different approaches are needed for patients with BMI or TSH levels when performing FNA.

Before multivariate analysis was done to evaluate clinical factors and malignant thyroid nodules, we performed the interaction test between BMI and TSH because a high BMI and TSH level have been shown to have positive association with thyroid malignancy. ${ }^{12,13,21)}$ It is well known that TSH is related to the energy metabolism of the body, ${ }^{22)}$ and perhaps due to the higher prevalence of hypothyroidism in obesity, there are early studies showing increased TSH levels in patients with high BMI. ${ }^{1423)}$ However, in our study, there was no significant association between BMl values and TSH levels, i.e., increasing BMI values and high TSH levels did not show significant association. Therefore, it was possible to include both BMI grade and $\mathrm{TSH}$ level as confounding factors in multivariate analysis to evaluate their association with malignant thyroid nodules.
The association between high BMI, TSH level, and risk of thyroid cancer cannot be explained in a simple manner. One proposed mechanism is explained by the role of leptin, which is an adipokine and known to be related to obese patients. ${ }^{24)}$ Elevated leptin levels will increase susceptibility to thyroid autoimmunity, which can lead to hypothyroidism and elevated TSH levels. ${ }^{25)}$ Another main mechanism is explained by insulin resistance in obese patients. ${ }^{26)}$ Insulin resistance, which is also related to diabetes mellitus, increases the insulin level in obese patients. Insulin can promote the secretion of regulatory factors including adipocytokines and $\mathrm{TSH}^{26)}$ In either of the suggested mechanisms, a high BMI will lead to a subsequent elevated TSH level and the increased amount of TSH stimulates the production of thyroid hormones and influences the growth and differentiation of thyroid cells. ${ }^{27)}$ The positive association between a high TSH level and increased risk of thyroid cancer $^{28)}$ and its aggressiveness ${ }^{29)}$ have been revealed. Recently, therapeutic strategy for thyroid cancer by means of controlling obesity with a drug has also been studied in mouse models. ${ }^{30}$ )

TSH stimulates the production of thyroid hormones and supports thyroid growth. ${ }^{27)}$ Although it is still controversial, some conclude that TSH receptor stimulation is related to the increased incidence and aggressiveness of thyroid cancer, ${ }^{29)}$ and there are reports that a high TSH level was significantly associated with thyroid malignancy. ${ }^{9,10)}$ On the other hand, suppression of TSH prevents the growth of thyroid nodules. ${ }^{31)}$ In our study, although it was not a significant factor in determining nodules for FNA, a high TSH level was significantly associated with malignancy in both univariate and multivariate analysis.

In a previous study, a low TSH level was associated with a lower risk of thyroid malignancy. ${ }^{32)}$ According to our results, low TSH levels were found to decrease risk of malignancy in both genders. There were $14.7 \%$ (318/2159) of benign nodules with suspicious US features in our study, therefore, if we can exclude those nodules for FNA, it will decrease the number of unnecessary FNAs. Further studies are needed to show the association between low TSH levels and lower risk of thyroid malignancy combined with US 
features of thyroid nodules.

Our results showing significant association between underweight (i.e., a low BMl grade) and thyroid malignancy in males is novel and not yet recognized. Most of the studies showing association between low BMl and cancer regards low BMl factor as a poor prognostic factor after surgery. ${ }^{33,34)}$ Still, in contrast to high $\mathrm{BMI}$ and cancer, there is a lack of report showing positive association between low BMI and malignancy. However, a recent study with a large cohort showed underweight was significantly associated with increased risk of breast cancer in premenopausal women. ${ }^{35)}$ Since number of males with low BMI was limited in our study, the relationship between thyroid malignancy and low BMI in males warrants further confirmation.

According to our results, smaller nodules were associated with malignancy in females. There are contradictory results regarding the thyroid nodule size and the risk of malignancy. Some studies show consistent result with ours, showing inverse relationship between the thyroid nodule size and risk of malignancy. ${ }^{36,37)}$ Still, other studies reported that a larger nodule is associated with higher risk of malignancy. ${ }^{38,39)}$ The higher malignancy rate in smaller nodules in our study could be related to higher rate of FNA on smaller nodules with suspicious US feature. There were some limitations to our study. First, there is an inevitable selection bias in our study. If a patient did not undergo surgery after FNA with inconclusive cytological results (indeterminate, suspicious for papillary thyroid carcinoma, or nondiagnostic), those nodules were excluded from the study subjects. Second, there is a possibility of false-negative or false-positive results for the thyroid nodules, as we included FNA cytological results, not surgical pathology, of malignant and benign nodules. However, we cannot attribute the potential misinterpretation of the results to false results on FNA, as previous studies conducted in our institution showed a relatively low rate of false-negative (1.9-3.2\%) or false-positive (1.9\%) results. ${ }^{16,40)}$ Third, there was a relatively small number of obese patients (3.23\%, $n=102)$ in our study population, which may limit the hazard assessment of the various factors.
Different results may be found in other study populations with a higher proportion of obese patients such as in western countries. Lastly, there were two different methods for TSH assay and associated two different reference levels for normal TSH level. According to a previous study, although the two assay methods well correlate with each other, IRMA was more sensitive than radioimmunoassay. ${ }^{41)}$

In conclusion, although there were more malignant thyroid nodules in patients with a high BMI grade or high TSH level, high BMI and high TSH levels were not significant factors in the determination of thyroid nodules for FNA when considered with US features.

\section{Conflict of Interest}

There is nothing to disclose.

\section{References}

1) Davies L, Welch HG. Increasing incidence of thyroid cancer in the United States, 1973-2002. JAMA 2006;295(18):2164-7.

2) Jung KW, Park S, Kong HJ, Won YJ, Lee JY, Park EC, et al. Cancer statistics in Korea: incidence, mortality, survival, and prevalence in 2008. Cancer Res Treat 2011;43(1):1-11.

3) Enewold L, Zhu K, Ron E, Marrogi AJ, Stojadinovic A, Peoples GE, et al. Rising thyroid cancer incidence in the United States by demographic and tumor characteristics, 1980-2005. Cancer Epidemiol Biomarkers Prev 2009;18(3):784-91.

4) American Thyroid Association (ATA) Guidelines Taskforce on Thyroid Nodules and Differentiated Thyroid Cancer, Cooper DS, Doherty GM, Haugen BR, Kloos RT, Lee SL, et al. Revised American Thyroid Association management guidelines for patients with thyroid nodules and differentiated thyroid cancer. Thyroid 2009;19(11):1167-214.

5) Meinhold CL, Ron E, Schonfeld SJ, Alexander BH, Freedman DM, Linet MS, et al. Nonradiation risk factors for thyroid cancer in the US Radiologic Technologists Study. Am J Epidemiol 2010;171(2):242-52.

6) Ogden CL, Yanovski SZ, Carroll MD, Flegal KM. The epidemiology of obesity. Gastroenterology 2007;132(6):2087-102.

7) Kitahara CM, Platz EA, Freeman LE, Hsing AW, Linet MS, Park Y, et al. Obesity and thyroid cancer risk among U.S. men and women: A pooled analysis of five prospective studies. Cancer Epidemiol Biomarkers Prev 2011;20(3):464-72.

8) Han JM, Kim TY, Jeon MJ, Yim JH, Kim WG, Song DE, et al. Obesity is a risk factor for thyroid cancer in a large, ultrasonographically screened population. Eur J Endocrinol 2013; 168(6):879-86.

9) Kim HK, Yoon JH, Kim SJ, Cho JS, Kweon SS, Kang HC. Higher TSH level is a risk factor for differentiated thyroid 
cancer. Clin Endocrinol (Oxf) 2013;78(3):472-7.

10) Haymart MR, Repplinger DJ, Leverson GE, Elson DF, Sippel RS, Jaume JC, et al. Higher serum thyroid stimulating hormone level in thyroid nodule patients is associated with greater risks of differentiated thyroid cancer and advanced tumor stage. J Clin Endocrinol Metab 2008;93(3):809-14.

11) Boelaert K, Horacek J, Holder RL, Watkinson JC, Sheppard MC, Franklyn JA. Serum thyrotropin concentration as a novel predictor of malignancy in thyroid nodules investigated by fine-needle aspiration. J Clin Endocrinol Metab 2006;91(11): 4295-301.

12) Diez JJ, Iglesias P. Relationship between thyrotropin and body mass index in euthyroid subjects. Exp Clin Endocrinol Diabetes 2011;119(3):144-50.

13) Fox CS, Pencina MJ, D'Agostino RB, Murabito JM, Seely $\mathrm{EW}$, Pearce EN, et al. Relations of thyroid function to body weight: cross-sectional and longitudinal observations in a community-based sample. Arch Intern Med 2008;168(6):587-92.

14) Rotondi M, Leporati P, La Manna A, Pirali B, Mondello T, Fonte R, et al. Raised serum TSH levels in patients with morbid obesity: is it enough to diagnose subclinical hypothyroidism? Eur J Endocrinol 2009;160(3):403-8.

15) Kwak JY, Kim EK, Kim HJ, Kim MJ, Son EJ, Moon HJ. How to combine ultrasound and cytological information in decision making about thyroid nodules. Eur Radiol 2009;19(8): 1923-31.

16) Kwak JY, Koo H, Youk JH, Kim MJ, Moon HJ, Son EJ, et al. Value of US correlation of a thyroid nodule with initially benign cytologic results. Radiology 2010;254(1):292-300.

17) Moon HJ, Kwak JY, Kim EK, Choi JR, Hong SW, Kim MJ, et al. The role of BRAFV600E mutation and ultrasonography for the surgical management of a thyroid nodule suspicious for papillary thyroid carcinoma on cytology. Ann Surg Oncol 2009;16(11):3125-31.

18) Yoon JH, Moon HJ, Kim EK, Kwak JY. Inadequate cytology in thyroid nodules: should we repeat aspiration or follow-up? Ann Surg Oncol 2011;18(5):1282-9.

19) World Health Organization Technical Report Series 894. Obesity: Preventing and managing the global epidemic. World Health Organization, Geneva, Switzerland, 2000.

20) Kim EK, Park CS, Chung WY, Oh KK, Kim DI, Lee JT, et al. New sonographic criteria for recommending fine-needle aspiration biopsy of nompalpable solid nodules of the thyroid. AJR Am J Roentgenol 2002;178(3):687-91.

21) Iacobellis G, Ribaudo MC, Zappaterreno A, Iannucci CV, Leonetti F. Relationship of thyroid function with body mass index, leptin, insulin sensitivity and adiponectin in euthyroid obese women. Clin Endocrinol (Oxf) 2005;62(4):487-91.

22) Kim B. Thyroid hormone as a determinant of energy expenditure and the basal metabolic rate. Thyroid 2008;18(2):141-4.

23) Reinehr T. Obesity and thyroid function. Mol Cell Endocrinol 2010;316(2):165-71.

24) Considine RV, Sinha MK, Heiman ML, Kriauciunas A, Stephens TW, Nyce MR, et al. Serum immunoreactive-leptin concentrations in normal-weight and obese humans. $N$ Engl J Med 1996;334(5):292-5.

25) Marzullo P, Minocci A, Tagliaferri MA, Guzzaloni G, Di
Blasio A, De Medici C, et al. Investigations of thyroid hormones and antibodies in obesity: Leptin levels are associated with thyroid autoimmunity independent of bioanthropometric, hormonal, and weight-related determinants. J Clin Endocrinol Metab 2010;95(8):3965-72.

26) Pazaitou-Panayiotou K, Polyzos SA, Mantzoros CS. Obesity and thyroid cancer: Epidemiologic associations and underlying mechanisms. Obes Rev 2013;14(12):1006-22.

27) Kimura T, Van Keymeulen A, Golstein J, Fusco A, Dumont JE, Roger PP. Regulation of thyroid cell proliferation by TSH and other factors: a critical evaluation of in vitro models. Endocr Rev 2001;22(5):631-56.

28) McLeod DS, Watters KF, Carpenter AD, Ladenson PW, Cooper DS, Ding EL. Thyrotropin and thyroid cancer diagnosis: a systematic review and dose-response meta-analysis. J Clin Endocrinol Metab 2012;97(8):2682-92.

29) Mazzaferri EL. Thyroid cancer and Graves' disease: the controversy ten years later. Endocr Pract 2000;6(2):221-5.

30) Park J, Kim WG, Zhao L, Enomoto K, Willingham M, Cheng SY. Metformin blocks progression of obesity-activated thyroid cancer in a mouse model. Oncotarget 2016;7(23): 34832-44.

31) Shi Y, Zou M, Farid NR. Expression of thyrotrophin receptor gene in thyroid carcinoma is associated with a good prognosis. Clin Endocrinol (Oxf) 1993;39(3):269-74.

32) Fiore E, Rago T, Provenzale MA, Scutari M, Ugolini C, Basolo F, et al. Lower levels of TSH are associated with a lower risk of papillary thyroid cancer in patients with thyroid nodular disease: thyroid autonomy may play a protective role. Endocr Relat Cancer 2009;16(4):1251-60.

33) Balakrishnan VS. Low BMI linked to worse colorectal cancer outcomes. Lancet Oncol 2015;16(16):e593.

34) Nakagawa T, Toyazaki T, Chiba N, Ueda Y, Gotoh M. Prognostic value of body mass index and change in body weight in postoperative outcomes of lung cancer surgery. Interact Cardiovasc Thorac Surg 2016;23(4):560-6.

35) Keinan-Boker L, Levine H, Derazne E, Molina-Hazan V, Kark JD. Measured adolescent body mass index and adult breast cancer in a cohort of 951,480 women. Breast Cancer Res Treat 2016;158(1):157-67.

36) Magister MJ, Chaikhoutdinov I, Schaefer E, Williams N, Saunders B, Goldenberg D. Association of thyroid nodule size and Bethesda class with rate of malignant disease. JAMA Otolaryngol Head Neck Surg 2015;141(12):1089-95.

37) Cavallo A, Johnson DN, White MG, Siddiqui S, Antic T, Mathew $\mathrm{M}$, et al. Thyroid nodule size at ultrasound as a predictor of malignancy and final pathologic size. Thyroid 2017;27(5):641-50.

38) Kamran SC, Marqusee E, Kim MI, Frates MC, Ritner J, Peters $\mathrm{H}$, et al. Thyroid nodule size and prediction of cancer. J Clin Endocrinol Metab 2013;98(2):564-70.

39) Hong MJ, Na DG, Baek JH, Sung JY, Kim JH. Impact of nodule size on malignancy risk differs according to the ultrasonography pattern of thyroid nodules. Korean J Radiol 2018;19(3):534-41.

40) Lee MJ, Hong SW, Chung WY, Kwak JY, Kim MJ, Kim EK. Cytological results of ultrasound-guided fine-needle aspiration 
BMI and TSH for Thyroid Nodule FNA

cytology for thyroid nodules: emphasis on correlation with sonographic findings. Yonsei Med J 2011;52(5):838-44.

41) Mehany MT, El Kolaly MT, Annyoub SM, Hassan SEM.

Immunoradiometric assay for the in-vitro determination of thyroid stimulating hormone in human serum and plasma using solid phase anti-TSH cellulose particles. J Radioanal Nucl Chem 2005;265(1):61-71. 
Appendix. Ultrasonographic features of the thyroid nodules

In our institution, the echogenicity of thyroid nodules was classified as followings; hyperechogenic (hyperechogenic compared to the normal thyroid gland), isoechogenic (isoechogenic compared to the normal thyroid gland), hypoechogenic (hypoechogenic compared to the normal thyroid gland but hyperechogenic to the surrounding strap muscle), and marked hypoechogenic (hypoechogenic compared to the surrounding strap muscle). Nodule margins are classified as well-circumscribed, microlobulated, and irregular. Calcifications are classified as microcalcification (calcification less than $1 \mathrm{~mm}$ ), macrocalcification (calcification $1 \mathrm{~mm}$ or larger), eggshell calcification, and no calcification. Nodule shape is classified as wider than tall (transverse diameter to the anteroposterior diameter ratio $<1$ ) and taller than wide (transverse diameter to the anteroposterior diameter ratio $\geq 1$ ). ${ }^{20}$ 